\title{
Phased Array of Phased Arrays (PAPA) Laser Systems Architecture
}

\author{
Paul F. McManamon \\ Air Force Research Laboratory \\ 2241 Avionics Circle, building 620 \\ Wright-Patterson AFB, OH 45433 \\ 937-255-4039 ext 4024 \\ paul.momanamon@wpafb.af.mil \\ William Thompson \\ Air Force Research Laboratory \\ Kirtland AFB, NM \\ 3550 Aberdeen Ave SE \\ Kirtland AFB, NM 87117-5776 \\ 505-846-2251 \\ william.thompson@kirtland.af.mil
}

\begin{abstract}
This paper introduces and analyzes a revolutionary laser system architecture capable of dramatically reducing the complexity of laser systems while simultaneously increasing capability. The architecture includes 3 major subsystems. The first is a phased array of laser sources. In this paper we discuss diode-pumped fiber lasers as the elements of the phased array, although other waveguide lasers can also be considered. The second provides wavefront control and electronics beam steering, as described in a WRG Baker award winning IEEE Proceedings article on "Optical Phased Array Technology". " The third is subaperture receiver technology. Combining these three technologies into a new laser systems architecture results in a system that has graceful degradation, can steer to as wide an angle as individual optical phased array sub-apertures, and can be scaled to high power and large apertures through phasing of a number of sub-apertures.
\end{abstract}

Diode-pumped fiber lasers are appealing as laser sources because they are electrically pumped, efficient, relatively simple, and scalable to significant power levels (over 100 Watts has been demonstrated from a single diode-pumped fiber laser. $\left.{ }^{2}\right)$ The fiber laser design also lends itself to integration into a phased array. Fiber lasers have been phased. ${ }^{3}$ Initial phasing demonstrations have been at low power and were conducted by taking a single source, dividing it into multiple fibers, then phasing them together. To develop this technology further we need to use independent fiber lasers or fiber amplifiers, seeded by a common source, and to increase laser power. As we increase laser power we will have to learn to cope with nonlinearities in the laser amplifiers.
Optical Phased Array technology has demonstrated steering over a 90-degree field of regard ${ }^{4}$, although this approach used additional optical components. If we use straightforward optical phased array beam steering without additional optics we can steer with high efficiency to about $1 / 3^{\text {rd }} \lambda / d$, where $d$ is the smallest individually addressable element. The $1 / 3^{\text {rd }}$ factor depends on the efficiency threshold. For example, if we use $1.5 \mu \mathrm{m}$ light, and $5 \mu \mathrm{m}$ center-to-center spacing we can steer with high efficiency to about plus or minus 6 degrees, or a field of regard of 12 degrees.

Lastly, we need to develop a sub-aperture receive technology. This can be a pupil plane receiver, an image plane receiver, or some combination of the approaches. When we have matured each individual technology, and combined them into new laser systems architecture, we will have the ability to build simpler and more capable laser systems. The vision for an integrated, phased array laser concept is to enable a new class of laser systems with significant advantages, including:

-- High efficiency, all electric laser source

- All waveguide beam transport

- Wavefront control at the sub-aperture level, enabling wavefront compensation, conformal apertures, and wide-angle electronic beam steering - Random access beam pointing over wide angles -- Multiple simultaneous beam generation and control

- Graceful degradation. ${ }^{1}$

\footnotetext{
${ }^{1}$ US Government work not protected by U.S. copyright
} 


\section{TABLE OF CONTENTS}

1. INTRODUCTION

2. OPTICAL PHASED ARRAY

TECHNOLOGY

3. PHASED ARRAY TRANSMITTERS

4. PHASED ARRAY RECEIVERS

5. CONCLUSIONS

6. BIOGRAPHIES

7. REFERENCES

\section{INTRODUCTION}

Laser systems can be used for a wide range of applications. All applications can be significantly simplified and enhanced using the architecture discussed here. Simplification should result in a much lower lifetime cost for these laser systems, and therefore more widespread application. Highenergy laser systems will of course have the largest gain in simplification. In a traditional HEL system architecture a high-bandwidth dynamic alignment system, coupled with sophisticated vibration isolation, is required to deal with jitter of the beam produced by the laser device and with the flexibility and vibration of the platform. A high-bandwidth, local-loop adaptive optics subsystem is required to deal with less-than-perfect beam quality produced by the laser device and with optical distortions due to heating of optical components by the laser beam. A separate target illuminator laser is required to meet signal-to-noise requirements for target tracking at high bandwidth, particularly to reject the jitter component of optical distortions introduced by atmospheric turbulence along the propagation path. A high-bandwidth, target-loop adaptive optics subsystem is required to deal with higherorder optical distortions introduced by atmospheric turbulence along the propagation path. An additional, separate beacon illuminator laser is required to meet signal-to-noise or point-ahead requirements for target-loop wavefront sensing at high bandwidth. Shared aperture tracking and target-loop wavefront sensing is required to eliminate the large non-common path errors that result from the use of a separate receive aperture.

To efficiently meet it's design objectives, any laser system must address similar, if less stressing, requirements and constraints as outlined above. This paper addresses an alternative approach - one that takes advantage of the unique characteristics of optical phased arrays.

Phasing an array of fiber lasers has the potential to develop physically distributed, high efficiency, electrically pumped lasers that can be scaled to high power. This could replace the laser in laser systems. However, to make a dramatic difference in laser systems we also have to dramatically simplify the beam control system, and build an associated receiver. Just replacing the laser does not provide all the performance benefit associated with a PAPA architecture. The requirements to produce coherent fiber laser array with both high power and good beam quality imply the capability to sense and control the phase of each element in the array and to expand and collimate the light from each element to produce a fully-filled, coherent, emitting aperture. These same capabilities can potentially contribute to some beam control functions, such as beam transport by fibers and piston phase control at the sub-aperture level for wavefront compensation and limited electronic beam steering.

Building on the inherent capabilities of phased array laser sources, it is conceptually possible to add additional technology elements in order to achieve the vision for an integrated phased array laser system. Specifically, the new technology elements needed are beam expansion so each fiber feeds a sub-aperture, sub-aperture tip and tilt wavefront control, and receive capabilities for target acquisition, wavefront sensing, target tracking, and laser beam pointing. Notionally, an integrated phased array laser system concept would be based on a phased array of diode-pumped fiber lasers, with each fiber feeding a crossed set of writable grating phased arrays (see figure 1). Figure 2 gives a potential specific implementation. 


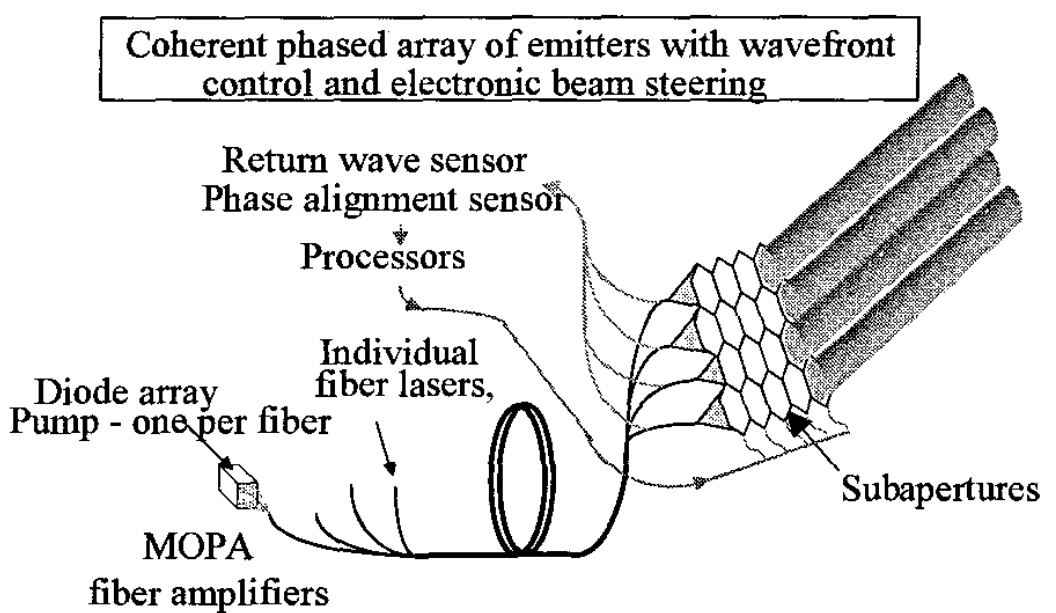

Figure 1 Phased Array of Phased Array Concept

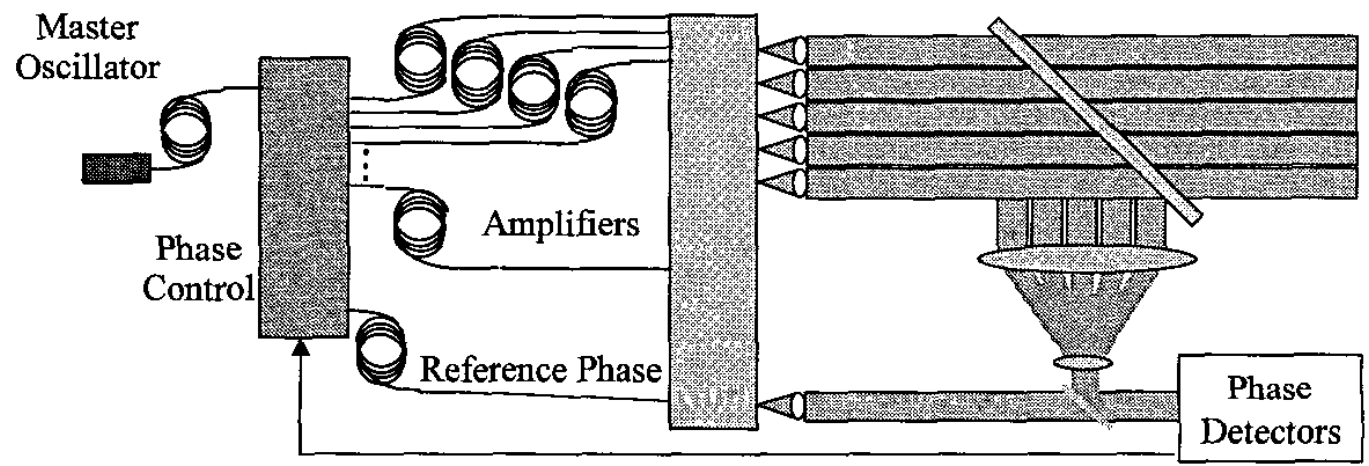

Figure 2 One-PAPA Concept Approach

The fiber laser geometry couples naturally with phased array beam control, allowing the expansion to large phased-array aperture diameters without the use of complex beam train optics. Phase patterns will be able to be imposed on each sub-aperture, and in addition piston phase changes could be imposed from sub-aperture to sub-aperture. The ability to incorporate electronic wavefront control at the sub-aperture level would provide the capability for large, random angle electronic beam steering from the phased array aperture. Piston phase shifts from one sub-aperture to the next may allow true time delay phased arrays. Since this is a phased array implementation it would no longer be necessary, when going from angle one to angle two, to pass through all the angles in between. It would also be possible to electronically adjust focus and to electronically generate multiple beams in various patterns.
As discussed above, phased array laser system concepts represent an entirely different approach to the design of a laser system. As you add fibers \& sub-apertures the laser power increases and the aperture size increases. This is a scaleable concept. You might have a 100-watt PAPA system consisting of 25 four-watt fibers each fed into a 2 $\mathrm{cm} \times 2 \mathrm{~cm}$ aperture. This results in a $10 \mathrm{~cm} \times 10$ $\mathrm{cm}$ aperture of 100 watts. You also could have 2500 -kilowatt fiber lasers, each feeding a $20 \mathrm{~cm} \mathrm{x}$ $20 \mathrm{~cm}$ aperture. This yields a 2.5 Megawatt laser system with a 10 meter $\times 10$-meter aperture. In concept neither of these systems has a complex beam control system.

If the attractive features of this concept can be realized in a practical and scalable system, it would have dramatic payoff across an exceptional range of laser system designs. 
The development of a phased array laser system conceptual design based on this vision offers significant challenges. Implementation approaches which best preserve the attractive features of the concept will require new technology approaches.

Technology development efforts are currently focused on individual optical phased array beam steerers. When applied at the sub-aperture level, this same technology may be applicable to the wavefront control and beam steering requirements for a phased array laser system. New ideas will probably be needed, however, to address specific phased array implementations and to achieve that goal of an integrated system concept.

A means to receive and process return signals from the target is necessary for target acquisition, tracking, aim point designation/control, and atmospheric compensation. While it is possible to use a separate, conventional aperture and sensor system to perform these functions, this extra aperture and beam train adds to the complexity (cost, weight, integration issues) of the overall laser system. It is thus highly desirable to develop techniques for collecting and sensing the return signals in a shared aperture as a function of the phased array itself. For some applications incoherent sensing at the sub-aperture level may be acceptable. Ultimately, however, coherent sensing will be required for many applications. This is a complex problem, and may have to be addressed through distributed sensing, such as pupil plane sensing and image reconstruction by computer. Optical layouts need to be investigated to determine the ideal method of doing receive as well as transmit. A straightforward approach may be considered that is essentially coherent beam combining in reverse, in which the return pulse is transmitted over a fiber to a focal plane array. Even in this approach we have the complexity that each return fiber must illuminate the focal plane array from a position that makes the light illuminating the FPA come from the same direction it would have come from if the sub-aperture was part of a monolithic receiver aperture.

\section{OPTICAL PHASED ARRAY TECHNOLOGY}

Optical phased array function is well described in reference 1. It can be used in each sub-aperture. Increasing the optical path delay across the aperture can provide this beam steering function, and then at least for a single wavelength, one can subtract $2 \pi$ phase shift, and repeat the increase in phase shift. The phase

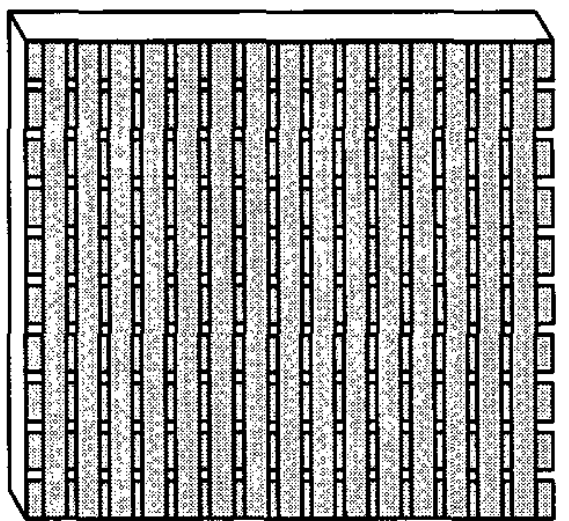

Figure 3 Crossed AZ / EL writable grating optical phased arrays

ramp across the aperture subtracts $2 \pi$ every time the optical path delay becomes larger than $2 \pi$. This can be accomplished with a set of step phase shifters, and we can use crossed AZ / EL writable gratings, as shown in figure 3

Figure 4 shows the phase ramp that can be placed across an aperture.

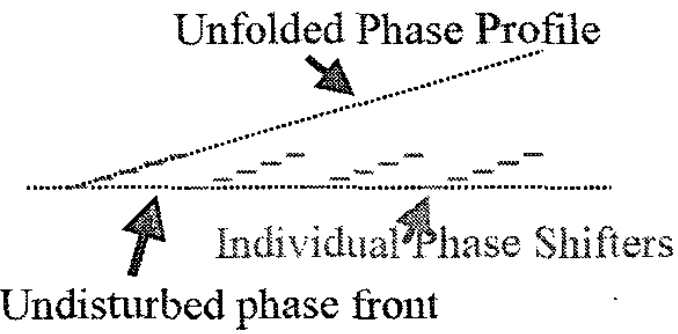

Figure 4 Phased Array phase Ramp with resets

One approach to generating the phase ramp is liquid crystals. Figure 5 shows a liquid crystal cell.

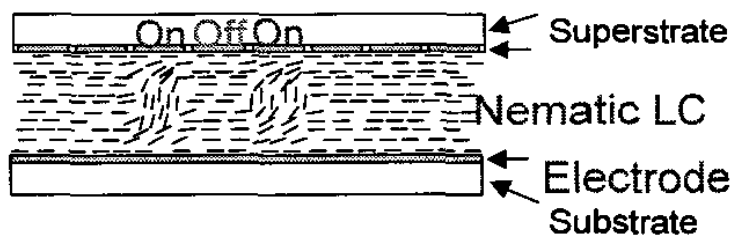

Figure 5 A Liquid Crystal Cell Cross section

When voltage is placed across the cell the optical path difference for a given polarization changes. This is a method of generating phase delays, up to a certain amount. The cell needs to be thicker to generate larger optical path delays, and 
therefore larger phase delays. As the cell becomes thicker the speed of the switch decreases as the square of the cell thickness. Therefore it makes sense to limit the required phase delay, and usually a modulo $2 \pi$ phase ramp approach is used. Figure 6 shows the graph of voltage vs. phase delay for a liquid crystal cell.

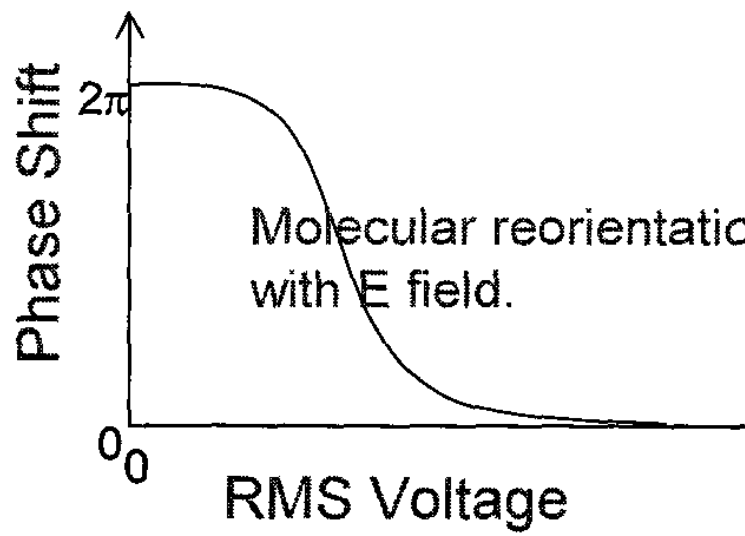

. Figure 6 Phase Shift vs., voltage for a liquid crystal cell

As mentioned in the abstract, to deflect a beam at large angles requires small phase retarding apertures. The rule of thumb used for phased array radar is that each phase retarding aperture should be one half wavelength center to center. If this is done, then it is possible to steer to 45 degrees deflection with approximately a cosine loss in steering efficiency. Unfortunately even if we can fabricate half wavelength center-to-center spacing electrodes it will be difficult to actually address the beam steered at this accuracy. It is not a major difficulty while proceeding up the phase ramp, for example, going from a 30-degree phase shift to a 60-degree phase shift. At the reset however we need to go from a nominal 360 phase shift to a nominal 0 degree phase shift. Fringing fields will be a problem for accurate addressing during reset. If we take a 0.2 birefringence liquid crystal material, the cell must be 5 wavelengths thick for a transmissive cell, or 2.5 wavelengths thick for a reflective cell. Traditionally fringing fields are about as wide as the cell is thick. This means for 0.2 birefringence our best addressing is about 2.5 wavelengths in reflective mode, or 5 wavelengths in transmissive mode, or a factor of 5 to ten away from the ideal phased array spacing. If we can use advanced liquid crystal with a 0.4 bi-refringence then we will be a factor of 2.5 to 5 away from ideal center-to-center spacing. It may be possible to push the rule of thumb concerning fringing fields.
If we can have fringing fields, which are not as wide as the cell is thick, we can get closer to good wide angle steering. To achieve half wavelength center-to-center spacing we would need to reduce fringing fields by the same factors discussed above. For example a factor of 5 for transmissive beam steering using a .4 birefringence. The liquid crystal's coherence length may also limit the smallest effective center-to-center spacing. This is an alternate potential limiting mechanism for fine addressing. Liquid crystal cells close each other do not readily change polarization.

For a straight -forward phased array the flaximum beam steering angle is:

$$
\theta=\frac{\lambda}{3 d}
$$

where the factor of 3 is a judgment factor depending on the efficiency threshold used. Using Eq. 1 we see the maximum deflection angle at this level of efficiency is plus or minus 4 degrees for .2 birefringence in a transmissive mode, and 7.5 degrees for .2 birefringence in a reflective mode - so long as liquid crystal special coherence does not limit the addressing. For .4 birefringence we could double these angles.

Because of the limitation currently associate with maximum steering angle other approaches are being implemented. These include holographic glass, ${ }^{5}$ lenslet arrays ${ }^{6}$, multiple prisms, ${ }^{7}$ and other approaches. These approaches are used to expand the angle provided for optical phased array beam steering so we can address a full field of regard of $>90$ degrees.

\section{PHASED ARRAY TRANSMITTERS}

A robust, integrated approach must be developed to ensure phase coherence and matching between all elements of the fiber laser array. A fiber array laser is shown in figure 7. As can be seen it starts with a single laser, and expands to multiple arrays of lasers.

The most promising current candidate for ensuring phase coherence among array elements is the master oscillator-power amplifier concept, but the scalability of this concept must be evaluated and demonstrated, both in terms of the number of phased array elements and the laser 
power from each element. The traditional approach to ensure phase matching at a given plane involves the use of bulk beam splitters and the use of interferometer techniques to interrogate the phase of each element at that point. This information is then used to adjust each element's phase to achieve phase matching. This approach, while feasible, is complex to implement in a real system and may make such attractive features as conformal apertures impossible. Work is needed to investigate and develop new, integrated approaches based on fiber optic sensing to interrogate the phase of each element. This work may leverage current true time-delay fiber based optical phased array development work both for microwave radar and for optical phased array development.

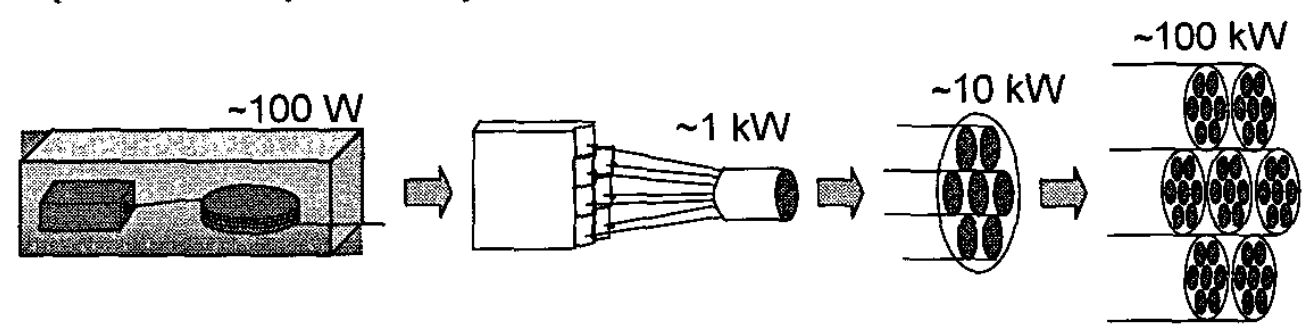

Figure 7 Phased Fiber Array Laser Architecture

New techniques will be needed to expand each beam from the core of each fiber laser $(\sim 10 \mu \mathrm{m}$ in diameter) to achieve a high fill factor at the final projecting phased array aperture in order to achieve reasonable beam quality. Optical phased array sub-aperture sizes might be anywhere from $1 \mathrm{~cm} \times 1 \mathrm{~cm}$ up to $20 \mathrm{~cm} \times 20$ $\mathrm{cm}$. Again, however, techniques that can be integrated with fiber lasers and concepts such as conformal apertures will have the most payoff. By having each fiber feed a phased array subaperture we can keep down the number of required fibers to be phased, and we can use the individual phased array sub-apertures to steer over wide angles.

\section{PHASED ARRAY RECEIVERS}

It is highly desirable to use the same aperture for transmit and receive functions. To accomplish this sub-aperture receive techniques need to be developed. Figure 8 and 9 show image plane and pupil plane imaging techniques. For an image plane technique we will have to bring each subaperture image to a focus from a spot as though it had been part of a larger aperture. Fiber position in illuminating the focal plane array will be critical. This will of course create fabrication problems. For image plane receive techniques we will need significant computational resources. It is possible that the best approach for PAPA may be a synergy between image plane and pupil plane imaging. Regardless additional development of sub-aperture receiver techniques will be required.

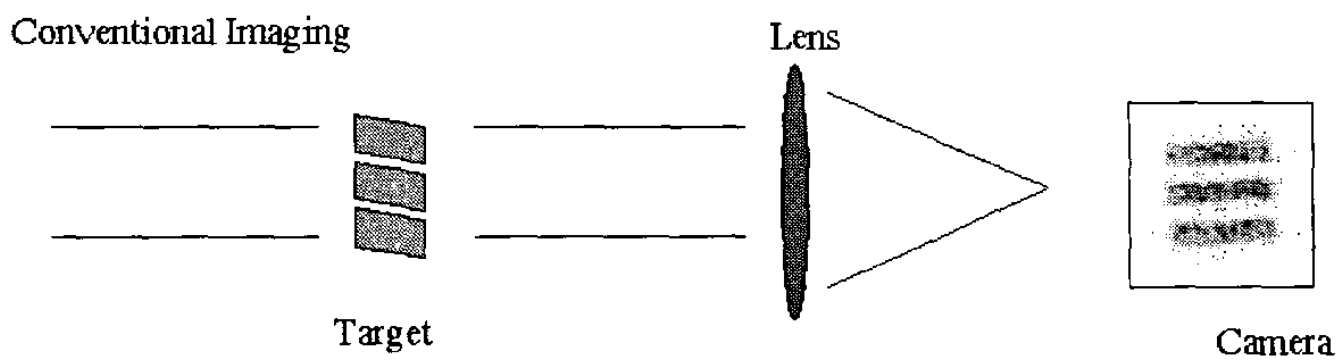

Figure 8a Conceptual Image Plane Receiver Diagram 


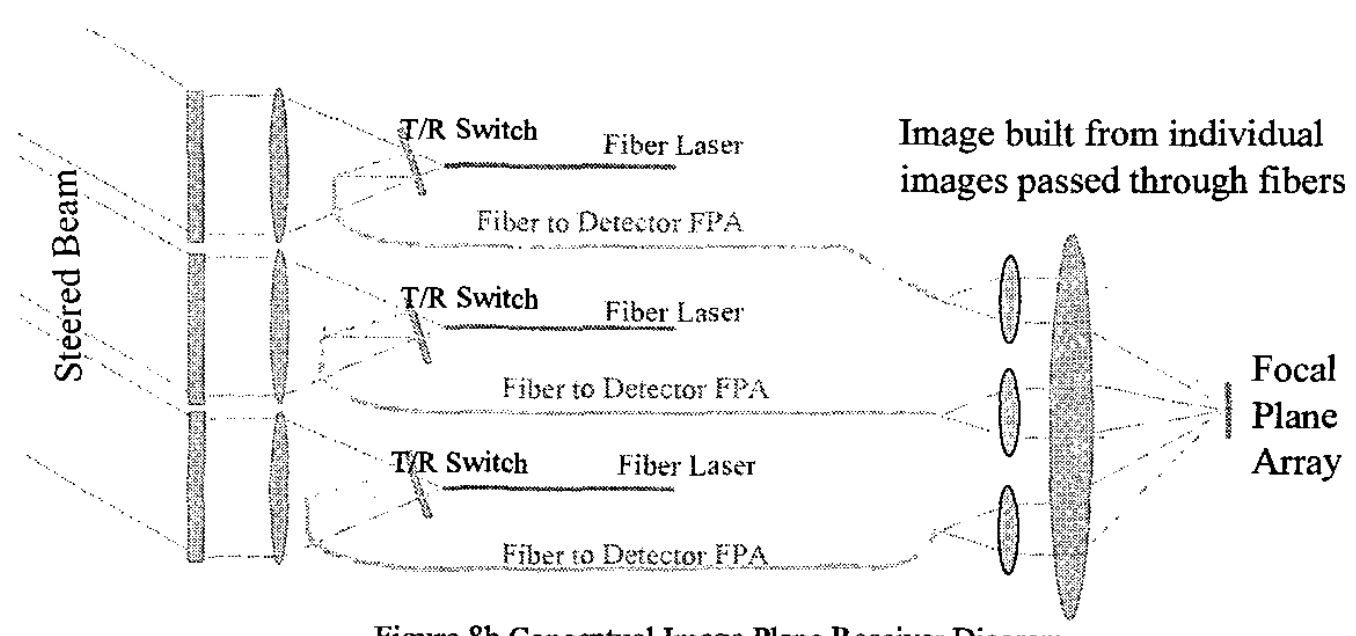

Figure 8 b Conceptual Image Plane Receiver Diagram

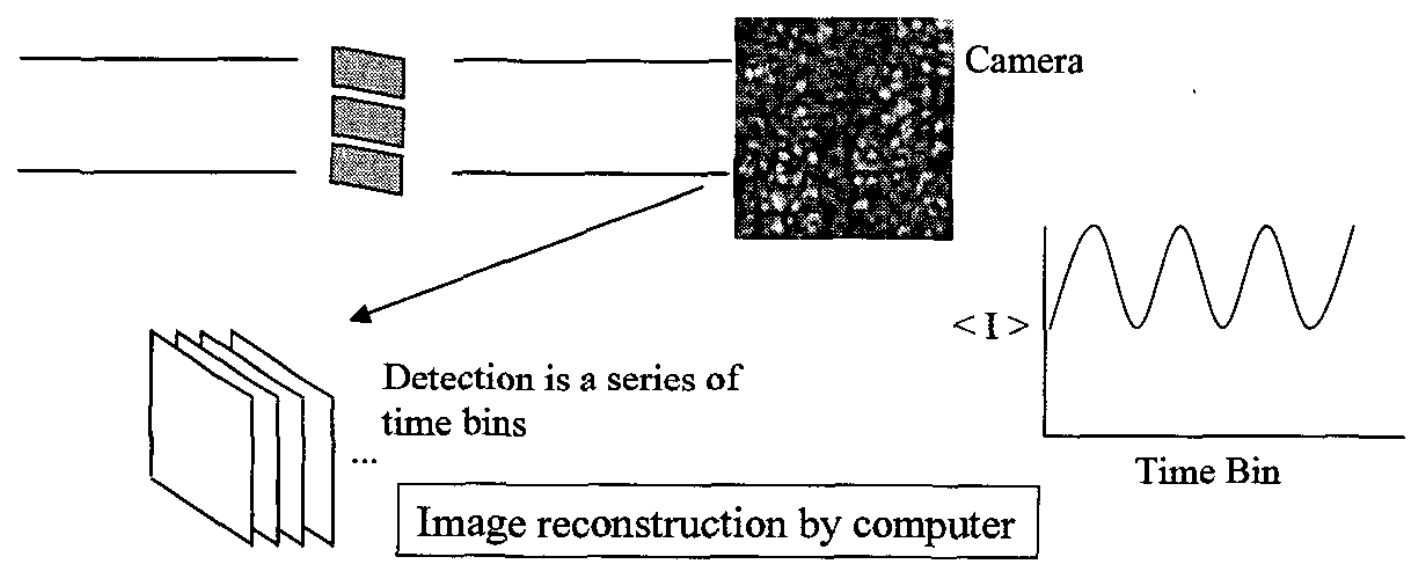

Figure 9 Pupil Plane Imaging Receiver Concepts

\section{CONCLUSIONS}

The ability to build a laser systems with a small number of components, no moving parts, and significant beam agility / flexibility will make future laser systems more attractive for all applications. Ongoing work in optical phased array beam steering and in arraying fiber lasers can be leveraged to make this happen.

Additional work will be required in sub-aperture receiver techniques, and of course in the integration of these three technologies into a single new laser systems architecture. Laser systems using this architecture will be developed for medium to high power. The larger systems will take significant development, but will have the most payoff in terms of systems simplification.

\section{BIOGRAPHIES}

Paul F. McManamon received his Ph.D. in Physics from Ohio State University in 1977. He has worked at Wright Patterson AFB since 1968, joining Wright laboratory in 1979 . His $\mathrm{PhD}$ was in laser physics. His primary work at in the laboratory has been in electro-optical sensors. He was primary author a paper entitled " Optical Phased Array Technology" that received the IEEE W.R.J. Baker award for the best paper in any IEEE referred journal or transaction. Recently he has emphasized laser radar sensors more than passive IR/EO sensors, but has research interests in both areas. He acted as Chief Scientist for the Avionics Directorate of Wright Lab for over two and a half years. He is currently senior scientist for Infrared Sensors, working in the Sensors Directorate of the Air Force Research Laboratory. He is a senior member of IEEE and a Fellow of SPIE. 
William E. Thompson is currently the Chief of the Strategic Development Branch, Directed Energy Directorate, Air Force Research Laboratory. He is responsible for the overall planning and programming for the Directed Energy Directorate. In previous positions within the Directorate, he has been the Program Manager for the Airborne Laser Technology program and, previously, Program Manger for the Ground-Based Laser Technology program. Mr. Thompson has also been the AFRL lead for the Space Optics and Lasers Integrated Technology Thrust Program, which integrated all AFRL activity into a common roadmap to address technology development and demonstration requirements for future operational space-based laser and optical systems for a range of missions. Starting in 1997, Mr.

Thompson was selected as the AFRL lead for the first phase of the Directed Energy Applications in Tactical Airborne Combat (DE ATAC) study, which investigated the feasibility and military utility of directed energy system concepts in a range of airborne tactical combat applications. Specifically relevant to this proposal, Mr. Thompson was also the deputy program manager for the Phased Integrated Laser-Optics Technology (PILOT) program, with the objective to develop and scale laser system concepts based on phased arrays of semiconductor laser diodes.

\section{REFERENCES}

${ }^{1}$ P. F. McManamon, T. A. Dorschner, D. C. Corkum, L. J. Friedman, D. S. Hobbs, M. K. O. Holz, S. Liberman, H. Nguyen, D. P. Resler, R. C. Sharp, and E. A. Watson, "Optical Phased Array Technology," Proc. IEEE 84(2), 268-298 (1996).

${ }^{2}$ V. Dominic, S. MacCormak, R. Waarts, S. Sanders,S. Bicknese, R. Dohle, E. Wolak, P.S. Yeh, \& E. Zucker, " 110 W Fibre Laser", Electronic Letters $8^{\text {th }}$ July 1999, Vol. 35 , No. 14

${ }^{3}$ Bob Rice, Private communication

${ }^{4}$ Terry Dorschner, private communications

${ }^{5}$ Terry Dorschner, private communication

${ }^{6}$ E. A. Watson, "Analysis of beam steering with decentered microlens arrays," Opt. Eng. 32(11), 2665-2670 (1993).

${ }^{7}$ Phil Bos, private communication 